\title{
Evaluation of heparin infusion rates in patients with intravenous drug misuse
}

\author{
Kenneth Barga ${ }^{1} \cdot$ Adam Smith $^{1} \cdot$ Mallory Faherty $^{2} \cdot$ Katherine Crawford $^{1}$ (i) \\ Accepted: 16 November 2021 / Published online: 22 November 2021 \\ (c) The Author(s), under exclusive licence to Springer Science+Business Media, LLC, part of Springer Nature 2021
}

\begin{abstract}
To evaluate the hypothesis that patients with a history of intravenous drug misuse (IVDM) initiated on weight-based heparin infusions require higher than expected infusion rates to achieve therapeutic activated partial thromboplastin time (aPTT). This study is a multicenter, retrospective chart review of patients with a history of IVDM who were admitted to an acute care site between 10/1/2015 and 9/30/2020 and treated with continuous heparin infusions. Patients were identified using ICD9 and ICD10 codes and included if they had a documented history of IVDM within the past six months. Variables of particular interest included: median heparin infusion rates to maintain therapeutic aPTT, average time to reach therapeutic aPTT, and International Society of Thrombosis and Haemostasis Criteria for moderate to severe bleeding. Of the 41 patients who met the inclusion and exclusion criteria, 39 achieved therapeutic aPTT while on a weight-based heparin infusion. All heparin infusions were initiated at a rate of $18 \mathrm{units} / \mathrm{kg} / \mathrm{hr}$ then titrated per institutional heparin infusion protocols. The mean time to therapeutic aPTT was $38.48 \mathrm{~h} \pm 26.4 \mathrm{~h}$ with a mean infusion rate of $27.64 \pm 7.14 \mathrm{units} / \mathrm{kg} / \mathrm{hr}$. To maintain therapeutic anticoagulation, infusion rates $150 \%$ higher than the initial rate were required. Of the 39 patients who achieved therapeutic aPTT, 85\% (33) met criteria for heparin resistance, defined as greater than 35,000 units of heparin daily. No statistical significance could be derived from this retrospective chart review as therapeutic heparin rates were evaluated in comparison to initial infusion rate, rather than a control group. The findings in this study demonstrate a possible clinical association of the reduced antithrombin activity previously described in opiate misusers. To efficiently achieve therapeutic anticoagulation, it may be appropriate to consider use of heparin antiXa monitoring in place of aPTT or utilization of increased initial heparin infusion rates.
\end{abstract}

Keywords Intravenous drug misuse $\cdot$ Heparin $\cdot$ Pharmacokinetics $\cdot$ Anticoagulation $\cdot$ Antithrombin

\section{Highlights}

Katherine Crawford

Katherine.Crawford@ohiohealth.com

Kenneth Barga

Kenneth.Barga2@ohiohealth.com

Adam Smith

Adam.Smith@ohiohealth.com

Mallory Faherty

Mallory.Faherty@ohiohealth.com

1 OhioHealth Riverside Methodist Hospital (Pharmacy Services), Columbus, OH, USA

2 OhioHealth Riverside Methodist Hospital (OhioHealth Research Institute), Columbus, OH, USA
- Implications of intravenous drug misuse (IVDM) on pharmacokinetic and pharmacodynamics properties of heparin are not well understood.

- The findings outlined in this study demonstrated increased heparin requirements in patients with a history of IVDM.

- Despite increased heparin dosing requirements, patients did not experience a clinically significant increase in moderate to severe bleeds as defined by the International Society of Thrombosis and Haemostasis (ISTH).

- Ideal future studies should include a larger sample size, compare findings to a control group, and be prospective in nature. 


\section{Background}

Unfractionated heparin is a parenteral anticoagulant that exerts its effects by inactivating thrombin and activated factor $\mathrm{X}$ within the coagulation cascade [1-3]. Through inactivation of thrombin, fibrin formation is inhibited, downstream platelets and clotting factors are not activated, and further clot formation is prevented. As such, heparin may be used to treat conditions such as pulmonary emboli (PE), deep vein thromboses (DVT), stroke, and other coagulopathies. To ensure therapeutic efficacy of heparin, aPTT is measured every six hours until two therapeutic aPTTs are obtained, and then aPTT is measured daily. At the care sites included in this study, therapeutic aPTT is defined as 68-104 s. (Supplemental Table 1 describes institutional heparin protocol).

Heparin may also be monitored using anti-Xa assays, which measure the ability of heparin antithrombin complex to inhibit activated coagulation factor X. Heparin anti-Xa is a more direct and less variable measure of heparin activity since it measures inhibition of only one enzyme. Additionally, heparin anti-Xa is less likely to be influenced by biologic factors. Despite these potential advantages, anti-Xa use may be limited by cost and laboratory availability [4-6].

In February 2020, a patient with history of IVDM received a continuous heparin infusion for the treatment of PE. In order to maintain therapeutic aPTT, the patient required heparin infusion rates of $38-52$ units $/ \mathrm{kg} / \mathrm{hr}$ totaling more than 100,000 units/day. This is notably higher than the threshold for heparin resistance, which is defined as requiring more than 35,000 units/day to reach therapeutic aPTT $[6,7]$.

Heparin resistance is most commonly caused by antithrombin deficiency but other causes may include congenital deficiencies, disseminated intravascular coagulation (DIC), increased heparin clearance levels (such as splenomegaly in liver disease), use of high doses of heparin during extracorporeal circulation, or use of asparaginase therapy. Heparin resistance has also been reported in critically ill patients with coronavirus disease 2019 (Covid-19) [6-11].

To date, there is no literature that evaluates continuous heparin infusion rates in patients with a history of IVDM; however, Ceriello and colleagues compared antithrombin activity and concentrations among 19 intravenous heroin misusers and 20 otherwise healthy volunteers [12]. A statistically significant decrease in antithrombin activity was observed in patients who misused heroin compared to healthy volunteers [12]. Patients with a history of IVDM also experienced a poor correlation between antithrombin concentrations versus antithrombin activity [12]. Given that the most common pathology for heparin resistance is antithrombin deficiency, we hypothesized that the reduced antithrombin activity observed in the IVDM population would manifest clinically as heparin resistance and that patients with history of IVDM would require higher than expected infusion rates to achieve and maintain therapeutic aPTT.

\section{Methods}

\section{Study design}

This multi-center, retrospective chart review evaluated patients with a documented history of IVDM who received treatment with a continuous weight-based heparin infusion for more than $48 \mathrm{~h}$.

All data was securely stored in REDCap (Research Electronic Data Capture) and subject to a second chart review to ensure data accuracy.

\section{Study population}

Subjects were identified using ICD9 (304.40, 304.50, 305.50, 305.60) and ICD10 (F11.10, F14.10, F15.10, F16.10) codes at seven acute care facilities admitted between 10/1/2015 and 9/30/2020. The study design flowchart and patient enrollment are shown in Fig. 1. A reproducible algorithm for identifying patients was created by an assigned
Fig. 1 Resultant sample population based on inclusion/exclusion criteria

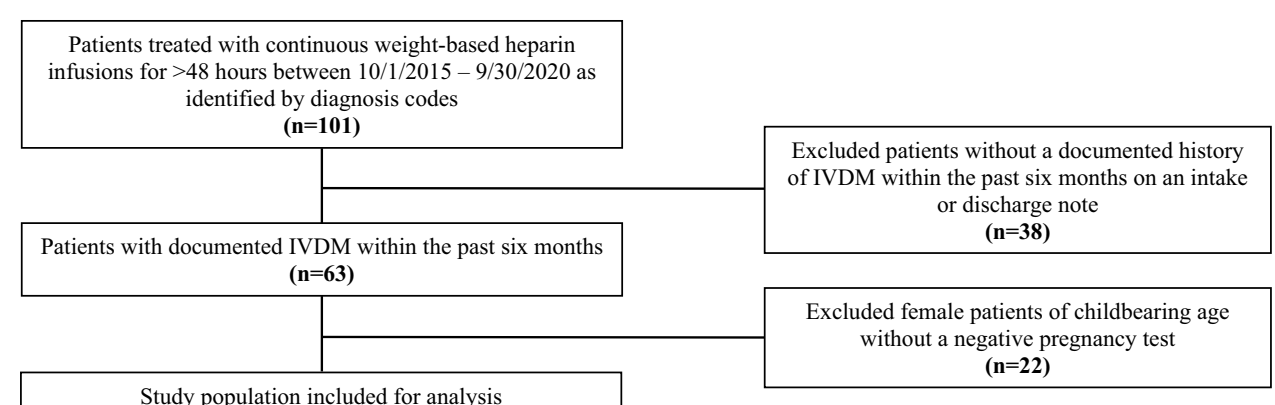

$(n=41)$ 
informaticist. This algorithm identified 101 potential subjects with at least one of the aforementioned diagnosis codes, age $>18$ years, and weight-based heparin infusions continued for more than $48 \mathrm{~h}$. Patients without a documented history of IVDM within the past six months in their admitting or discharge progress notes were excluded $(n=38)$. Of the 63 potential subjects that remained, 22 patients were females of childbearing age without a negative pregnancy test on file. Once removed, this resulted in a sample size of 41 patients.

\section{Inclusion criteria}

All patients age 18 years or older with a recent documented history (within the past six months) of IVDM who received treatment with a weight-based heparin infusion between 10/1/2015 and 9/30/2020 were included in this study. For the purposes of streamlined data collection, only patients who presented to an acute care facility with an implemented, shared electronic medical record on or before $10 / 1 / 2015$ were included.

\section{Exclusion criteria}

Pregnant females and women of child-bearing age without a negative pregnancy test on file were excluded. Patients were also excluded if the heparin infusion was non-continuous (i.e., interrupted outside of what would have been required of the weight-based heparin infusion protocol) or if the heparin infusion was not continued for at least 48 consecutive hours. Patients who were initiated on a heparin infusion at a facility outside of the seven identified acute care sites were excluded as the research team could not confirm that a similar weight-based heparin protocol was used.

\section{Study outcomes}

The primary outcome of this retrospective chart review was to describe therapeutic heparin infusion rates in patients with recent (within the past six months) documented history of IVDM for the treatment of all labeled indications. To describe this aim, the lowest, highest, and maintenance infusion rates were recorded for all patients who reached a therapeutic activated partial thromboplastin time (aPTT).

Secondary outcomes looked to determine the average time to reach therapeutic aPTT in this patient population and to identify patients who have suffered moderate to severe bleeds secondary to increased heparin infusion rates. To help define the secondary safety outcome, the International Society of Thrombosis and Haemostasis (ISTH) criteria were utilized [13], which noted fatal bleeds, bleeds in a critical area, and bleeds that resulted in a decrease in hemoglobin by more than $2 \mathrm{~g} / \mathrm{dL}$ and/or required blood transfusions.

\section{Statistical analysis}

All data was analyzed utilizing Excel and SPSS. This was a descriptive study; all variables were summarized utilizing appropriate descriptive statics. Continuous variables were summarized with means and standard deviations. Categorical variables were summarized with counts and percentages.

\section{Results}

\section{Demographic outcomes}

In total, 41 patients met the inclusion and exclusion criteria previously described. All patient demographic information is located in Table 1.

\section{Clinical outcomes}

Table 2 and Fig. 2 represent heparin infusion requirements among the 41 patients included in this study. As the table indicates, 39 of the 41 patients achieved therapeutic aPTT. Average time to therapeutic anticoagulation was 35.5 (SD: \pm 26.4 ) hours. To maintain therapeutic aPTT, an average infusion rate of $27.6(\mathrm{SD}: \pm 7.1) \mathrm{units} / \mathrm{kg} / \mathrm{hr}$ was required. Maintenance infusion rate, as defined by this study, is the heparin infusion rate for which patients maintained therapeutic aPTT for the longest duration. The mean maintenance infusion rate for patients in this study was $150 \%$, or 9.6 units $/ \mathrm{kg} / \mathrm{hr}$ more than the initial heparin infusion rate. When median infusion rate was multiplied over a $24 \mathrm{~h}$ period, $85 \%$ of patients with therapeutic aPTT (33) met criteria for heparin resistance, defined as greater than 35,000 units of heparin daily.

\section{Safety outcomes}

The International Society on Thrombosis and Haemostasis (ISTH) defines major bleeding in non-surgical patients as symptomatic presentation coupled with one of the following criteria: (1) fatal bleed, (2) symptomatic bleed in a critical area, and/or (3) bleed associated with a decrease in hemoglobin level of more than $2 \mathrm{~g} / \mathrm{dL}$ or requiring blood transfusions of two or more units of whole blood/red blood cells [9]. Critical areas are defined as intracranial, intraspinal, intraocular, retroperitoneal, intra-articular, intra-pericardial, or intramuscular with compartment syndrome [9]. Of the 41 patients included in this study, none suffered a fatal bleed or a symptomatic bleed in a critical area. 
Table 1 Summary of baseline patient demographics

\begin{tabular}{|c|c|}
\hline Patient demographics & $\begin{array}{l}\text { Heparin infusion } \\
\text { in IVDM }(n=41)\end{array}$ \\
\hline Age (years) $\pm \mathrm{SD}$ & $35 \pm 9.4$ \\
\hline \multicolumn{2}{|l|}{ Gender } \\
\hline Male & $18(43.9 \%)$ \\
\hline Female & $23(56.1 \%)$ \\
\hline Total body weight $(\mathrm{kg}) \pm \mathrm{SD}$ & $74.2 \pm 21.6$ \\
\hline \multicolumn{2}{|l|}{ Body mass index $\left(\mathrm{kg} / \mathrm{m}^{2}\right)$} \\
\hline$<18.5$ & $4(9.8 \%)$ \\
\hline $18.5-24.9$ & $14(34.1 \%)$ \\
\hline $25-29.9$ & $13(31.7 \%)$ \\
\hline $30-34.9$ & $8(19.5 \%)$ \\
\hline $35-39.9$ & $1(2.4 \%)$ \\
\hline$\geq 40$ & $1(2.4 \%)$ \\
\hline \multicolumn{2}{|l|}{$\mathrm{CrCl}(\mathrm{mL} / \mathrm{min})$} \\
\hline$<15$ or Dialysis & $0(0 \%)$ \\
\hline $15-29.9$ & $3(7.3 \%)$ \\
\hline $30-59.9$ & $1(2.4 \%)$ \\
\hline $60-89.9$ & $6(14.6 \%)$ \\
\hline$\geq 90$ & $31(75.6 \%)$ \\
\hline \multicolumn{2}{|l|}{ Indication for heparin infusion } \\
\hline Acute deep vein thrombosis (DVT) & $20(49 \%)$ \\
\hline Acute pulmonary embolism (PE) & $14(34 \%)$ \\
\hline History of DVT/PE & $7(17 \%)$ \\
\hline \multicolumn{2}{|c|}{ Proximity of IVDM to hospital admission } \\
\hline$<48 \mathrm{~h}$ & $18(43.9 \%)$ \\
\hline$\leq 1$ week & $11(26.8 \%)$ \\
\hline$>1$ week & $6(14.6 \%)$ \\
\hline$<1$ month & $6(14.6 \%)$ \\
\hline \multicolumn{2}{|l|}{ Urine toxicology results } \\
\hline Positive & $29(70.7 \%)$ \\
\hline Opiates & $14(34 \%)$ \\
\hline Oxycodone & $4(9.8 \%)$ \\
\hline Cocaine & $10(24.4 \%)$ \\
\hline Amphetamines & $12(29.3 \%)$ \\
\hline Benzodiazepines & $2(4.9 \%)$ \\
\hline Barbituates & $1(2.4 \%)$ \\
\hline Tetrahydrocannabinol (THC) & $5(12.2 \%)$ \\
\hline Tricyclic antidepressants & $1(2.4 \%)$ \\
\hline Fentanyl & $1(2.4 \%)$ \\
\hline Methadone & $1(2.4 \%)$ \\
\hline Negative & $4(9.7 \%)$ \\
\hline Not obtained & $8(19.5 \%)$ \\
\hline
\end{tabular}

One patient did experience a gastrointestinal bleed that resulted in a hemoglobin decrease of more than $2 \mathrm{~g} / \mathrm{dL}$, which qualified as a major bleeding event. This data is summarized in Table 3.
Table 2 Summary of heparin infusion rates in patients with history of IVDM

\begin{tabular}{ll}
\hline Heparin infusion rates in patients with history of IVDM $(\mathrm{n}=41)$ \\
\hline Number of patients who achieved therapeutic aPTT $(\%)$ & $39(95.1 \%)$ \\
Time to reach therapeutic aPTT, mean $(\mathrm{hr})$ & $38.5 \pm 26.4$ \\
Initial infusion rate, mean (units/kg/hr) & $18 \pm 0$ \\
Lowest infusion rate when therapeutic, mean (units $/ \mathrm{kg} /$ & $25 \pm 7.8$ \\
hr) & \\
Highest infusion rate when therapeutic, mean (units/ & $28.7 \pm 6.6$ \\
$\mathrm{~kg} / \mathrm{hr}$ ) & \\
Maintenance infusion rate, mean (units/kg/hr) & $27.6 \pm 7.1$ \\
\hline
\end{tabular}

\section{Discussion}

Our study aimed to address a knowledge gap in the literature surrounding altered heparin pharmacokinetics in patients with history of IVDM. A prior study by Ceriello and colleagues found no difference in antithrombin plasma concentration between heroin users and control group; however, antithrombin activity was demonstrated to be significantly decreased in heroin users. As of the date of publication, there are no studies that evaluate the impact of IVDM on heparin infusion requirements. As previously described, the IVDM patients in our study required heparin infusion rates over $150 \%$ of the initial recommended infusion rate. The increase in heparin dosing requirements is most likely explained by reduced antithrombin activity previously demonstrated in IVDM [12].

Antithrombin is a serine protease inhibitor which serves as the primary inhibitor of thrombin, but also inactivates factors IXa, Xa, XIa, and XIIa of the coagulation cascade [9]. As heparin depends on presence of antithrombin to exert therapeutic effect, patients with antithrombin deficiency often require higher than expected heparin infusion rates to achieve therapeutic anticoagulation. Additionally, illicit drug users have demonstrated increased secretion of acute phase reactants such as C-reactive protein (CRP) and serum amyloid A (SAA) [14]. Since some of the heparin-binding proteins are also acute-phase reactants, nonspecific binding to these proteins may contribute to variability of anticoagulant response and heparin resistance in patients with IVDM [15].

Laboratory monitoring of heparin may serve as another explanation for the increased heparin infusion rates observed in this study. A study by Levine et al. demonstrated superiority of heparin anti-Xa assays in heparin resistant patients (requiring greater than 35,000 units of heparin daily) [16]. Levine et al. also demonstrated significantly reduced amounts of heparin in patients monitored by anti-Xa assays [16]. When heparin resistance is a concern, anti-Xa assays can be used to measure anticoagulation from heparin, with a target of 0.3 to 0.7 IU per milliliter [4-6]. As this study was retrospective, anti-Xa measurements were not available for 
Fig. 2 Heparin infusion rate and time to therapeutic aPTT for all patients who achieved therapeutic aPTT
Heparin infusion rate and time to therapeutic aPTT

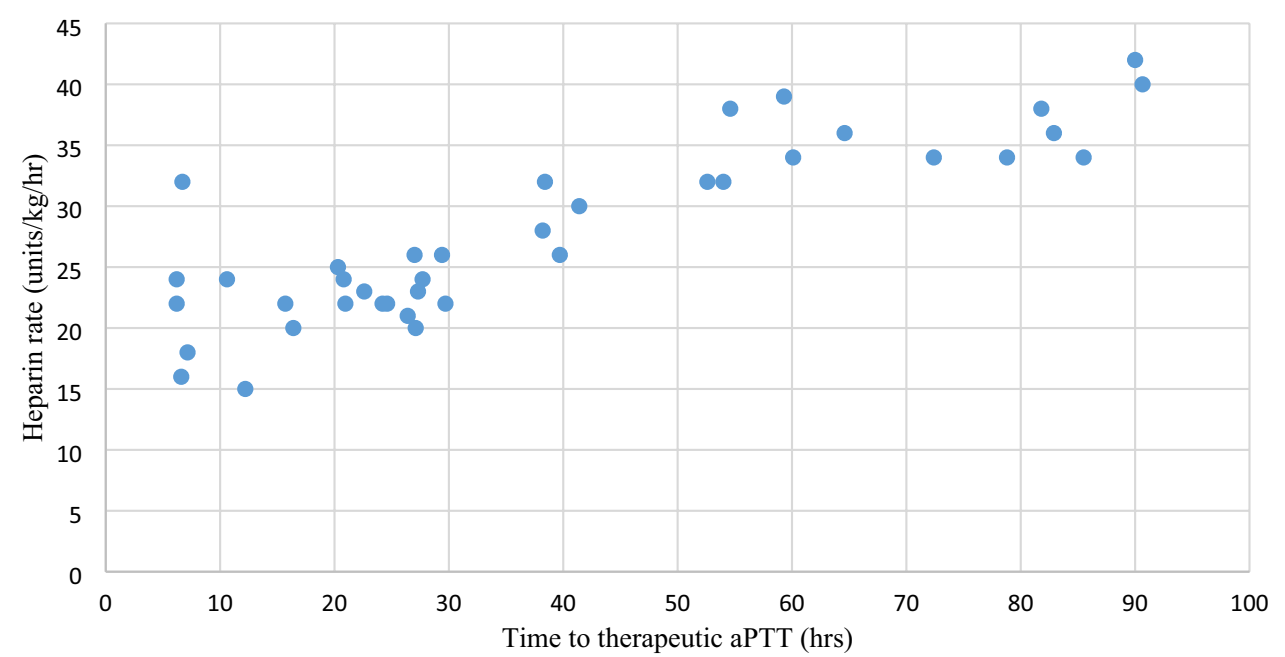

Table 3 Assessment of moderate to severe bleeds as defined by ISTH criteria

\begin{tabular}{lll}
\hline Assessment of ISTH criteria $(\mathrm{n}=41)$ & & \\
\hline & Yes & No \\
\hline Fatal bleed & 0 & 41 \\
Symptomatic bleed in a critical area & 0 & 41 \\
Bleed associated with a decrease in Hgb $>2$ g/dL & 1 & 40 \\
and/or requiring blood transfusions & & \\
\hline
\end{tabular}

analysis, as aPTT is the standard laboratory utilized by our institution for heparin monitoring.

Previous literature has shown altered vancomycin and aminoglycoside pharmacokinetics in the IVDM population as well, where increased doses were required to reach therapeutic concentrations $[17,18]$. These studies postulated that increased requirements may be secondary to enhanced clearance via altered filtration and secretory clearance. Heparin is similar to aminoglycosides and vancomycin in that it is a large molecule; however, it is only partially eliminated through renal excretion, so alterations in renal function are likely not the sole contributor for the clinical findings observed in our study.

The retrospective design of our study limited our ability to control for external confounding factors. Also, heparin anti-Xa levels were unavailable for interpretation as aPTT is the standard heparin monitoring lab at our institution. Documentation of IVDM, and therefore patient inclusion, was dependent upon patient reporting in a population that may not be forthcoming about their illicit substance use. Generalizability of our study may be limited as although performed at multiple centers, each campus is within the same health system and primarily located within central
Ohio. Furthermore, external validity may be limited by use of an institution specific dosing calculator for determination of heparin drip titration in accordance to aPTT. Lastly, our study lacked a non-intravenous drug misusing control group, opting to use the initial heparin rate as a comparator.

Given the continued opioid epidemic and growth of intravenous drug misuse in recent years, this study sought to address a lack of literature in the IVDM population. Current weight-based heparin infusion protocols do not differentiate between intravenous drug misusers and their non-using counterparts. This lack of distinction can potentially lead to a delay in therapeutic anticoagulation. Future studies are required to validate the findings of our evaluation. Ideally, studies would include a larger sample size with a non-intravenous drug misusing control group. These studies should be prospective to prevent confounding factors, limit discrepancies in documentation, and potentially differentiate between the substances that were misused. Prospective studies should rule out other causes of heparin resistance such as increased levels of factor VIII or fibrinogen, deficiencies of antithrombin, and binding of acute phase proteins to heparin. Additionally, further evaluation on effect of other factors such as route of drug administration and substance of abuse would further characterize heparin pharmacokinetic changes in this population.

\section{Conclusion}

The findings in this study demonstrate a possible clinical association of the reduced antithrombin activity previously described in opiate misusers. To efficiently achieve therapeutic anticoagulation, it may be appropriate to consider use of 
heparin antiXa monitoring in place of aPTT or utilization of increased initial heparin infusion rates.

Supplementary Information The online version contains supplementary material available at https://doi.org/10.1007/s11239-021-02615-z.

Acknowledgements Jason Harris, CPhT.

Author contributions Conceptualization: [KC]; Methodology: [KC, $\mathrm{KB}, \mathrm{MF}]$; Data collection: [KB, MF]; All authors contributed to data analysis. Writing —original draft preparation: $[\mathrm{KB}]$; Writing - review and editing: [KC, AS]; Resources: [KC, KB]; Supervision: [KC, AS]. All authors read and approved the final manuscript.

Funding No funds, grants, or other support was received.

Data availability All data is securely stored using Research Electronic Data Capture (REDCap).

Code availability Not applicable.

\section{Declarations}

Conflict of interest None of the four authors, Kenneth Barga, Katherine Crawford, Mallory Faherty, nor Adam Smith have any conflicts of interest to declare.

Ethical approval Ethical approval was waived by the OhioHealth Investigational Review Board (IRB) in view of the retrospective nature of the study and all the procedures being performed were part of the routine care.

\section{References}

1. Hirsh J, Anand SS, Halperin JL et al (2001) Mechanism of action and pharmacology of unfractionated heparin. Arterioscler Thromb Vasc Biol 21:1094-1096. https://doi.org/10.1161/hq0701.093686

2. Hirsh J, Warkentin TE, Shaughnessy SG et al (2001) Heparin and low-molecular-weight heparin: mechanisms of action, pharmacokinetics, dosing, monitoring, efficacy, and safety. Chest 119:64S-94S

3. Boneu B, Caranobe C, Sie P (1990) Pharmacokinetics of heparin and low molecular weight heparin. Baillieres Clin Haematol 3:531-544. https://doi.org/10.1016/s0950-3536(05)80017-4

4. Samuel S, Allison TA, Sharaf S et al (2016) Antifactor Xa levels vs activated partial thromboplastin time for monitoring unfractionated heparin. J Clin Pharm Ther 41(5):499-502. https://doi. org/10.1111/jcpt. 12415

5. Guervil DJ, Rosenberg AF, Winterstein AG et al (2011) Activated partial thromboplastin time versus antifactor Xa heparin assay in monitoring unfractionated heparin by continuous intravenous infusion. Ann Pharmacother 45(7-8):861-868. https://doi.org/10. 1345/aph.1Q161

6. Levy JH, Connors JM (2021) Heparin resistance- clinical perspectives and management strategies. N Engl J Med 385:826-832. https://doi.org/10.1056/NEJMra2104091

7. Durrani J, Malik F, Ali N et al (2018) To be or not to be a case of heparin resistance. J Community Hosp Intern Med Perspect 8(3):145-148. https://doi.org/10.1080/20009666.2018.1466599

8. Sniecinski RM, Bennett-Guerrero E, Shore-Lesserson L (2019) Anticoagulation management and heparin resistance during cardiopulmonary bypass: a survey of society of cardiovascular anesthesiologists members. Anesthesia Analgesia https://doi.org/10. 1213/ANE.0000000000003981

9. Hsu E, Moosavi L (2020) Biochemistry, antithrombin III. StatPearls. Online publication 2020 Sep; updated 2021 Jan.

10. Moll S (2009) Antithrombin deficiency. National Blood Clot Alliance. https://www.stoptheclot.org/news/antithrombin-deficiency/. Accessed 7 Sep 2021.

11. Bauer K (2018) Antithrombin deficiency. Rare Disease Database. https://rarediseases.org/rare-diseases/antithrombin-deficiency/. Accessed 7 Sep 2021

12. Ceriello A, Russo PD, Curcio F et al (1984) Depressed antithrombin III biological activity in opiate addicts. J Clin Pathol 37:1040-1042

13. Schulman S, Kearon C (2005) Subcommittee on control of anticoagulation of the scientific and standardization committee of the international society on thrombosis and haemostasis. Definition of major bleeding in clinical investigations of antihemostatic medicinal products in non-surgical patients. J Thromb Haemost 3(4):692-694 https://doi.org/10.1111/j.1538-7836.2005.01204.x

14. Samikkannu T, Rao K, Arias AY et al (2013) HIV infection and drugs of abuse: role of acute phase proteins. J Neuroinflammation 10:113. https://doi.org/10.1186/1742-2094-10-113

15. Young E, Podor TJ, Venner T, Hirsh J (1997) Induction of the acute-phase reaction increases heparin-binding proteins in plasma. Arterioscler Thromb Vasc Biol 17(8):1568-1574. https://doi.org/ 10.1161/01.atv.17.8.1568

16. Levine MN, Hirsh J, Gent M, et al. (1994) A randomized trial comparing activated thromboplastin time with heparin assay in patients with acute venous thromboembolism requiring large daily doses of heparin. Arch Intern Med 154:49-56 https://doi.org/10. 1001/archinte.154.1.49

17. King CH, Creger RJ, Ellner JJ (1985) Pharmacokinetics of tobramycin and gentamicin in abusers of intravenous drugs. Antimicrob Agents Chemother 27(3):285-290 https://doi.org/10.1128/ aac.27.3.285

18. Rybak MJ, Albrecht LM, Berman JR, et al. (1990) Vancomycin pharmacokinetics in burn patients and intravenous drug abusers. Antimicrob Agents Chemother 34(5):792-795 https://doi.org/10. 1128/aac.34.5.792

Publisher's Note Springer Nature remains neutral with regard to jurisdictional claims in published maps and institutional affiliations. 